\title{
Los derechos humanos frente al espejo. Narrativas de mujeres sin hogar en el ejercicio de sus capacidades
}

Human Rights in front of the Mirror. Narratives of Homeless Women in the Exercise of Their Capacities

Les droits humains devant le miroir. Récits de femmes sans-abri dans l'exercice de leurs capacités

Nuria Cordero-Ramos y Manuel Muñoz-Bellerin

(2) OpenEdition

Journals

Edición electrónica

URL: https://journals.openedition.org/rccs/12407

DOI: $10.4000 /$ rccs. 12407

ISSN: 2182-7435

Editor

Centro de Estudos Sociais da Universidade de Coimbra

Edición impresa

Fecha de publicación: 1 diciembre 2021

Paginación: 47-68

ISSN: 0254-1106

Referencia electrónica

Nuria Cordero-Ramos y Manuel Muñoz-Bellerin, «Los derechos humanos frente al espejo. Narrativas de mujeres sin hogar en el ejercicio de sus capacidades», Revista Crítica de Ciências Sociais [En línea] 126 | 2021, Publicado el 27 enero 2022, consultado el 29 enero 2022. URL: http:// journals.openedition.org/rccs/12407 ; DOI: https://doi.org/10.4000/rccs.12407 


\section{NURIA CORDERO-RAMOS, MANUEL MUÑOZ-BELLERIN}

\section{Los derechos humanos frente al espejo. Narrativas de mujeres sin hogar en el ejercicio de sus capacidades}

Partiendo de las consecuencias que provoca el sinhogarismo en las mujeres y teniendo presente el reconocimiento de los derechos humanos, se analizan las experiencias de las propias protagonistas: mujeres sin hogar que están viviendo en centros de acogida de la ciudad de Sevilla (España). Se toman como referencia las narrativas de las propias protagonistas como un recurso alegórico de espacio para narrar experiencias de resistencia. Estas narraciones fueron relatadas durante la realización de una serie de talleres donde las mujeres van compartiendo emociones y vivencias gracias a la apropiación personal y colectiva del lenguaje artístico. El trabajo pretende destacar la dimensión creativa del teatro aplicado como herramienta que permite crear espacios que garanticen la dignidad de las mujeres sin hogar, ofreciéndoles la oportunidad de mostrar sus capacidades al conjunto de la sociedad.

Palabras clave: derechos de la mujer; España; instituciones de acogimiento; sin hogar; violación de los derechos humanos.

Palavras-chave: direitos da mulher; Espanha; instituições de acolhimento; sem-abrigo; violação dos direitos humanos.

\section{Introducción}

A lo largo de este artículo se analiza la situación en la que están inmersas las mujeres sin hogar (en adelante MSH) tomando prestadas las voces de mujeres que hablan de sus experiencias como fuente de un espacio biográfico que Arfuch declara como espacio común a partir de las subjetividades (2018: 19). Estas narraciones se han obtenido a través del trabajo de campo realizado en el período comprendido entre los años 2016-2019 en diversos 
centros de acogida (en adelante CA) ${ }^{1}$ de la ciudad de Sevilla, en España, como parte de una investigación llevada a cabo por los autores en la que se aborda el teatro como estrategia de memoria activa (Segato, 2019) y derechos humanos en personas sin hogar.

El texto comienza con una aproximación al sinhogarismo y los efectos que este fenómeno causa en las mujeres en Europa y España, con el objetivo de entender las implicaciones que tiene la opresión y la dominación desde la perspectiva de género y los derechos humanos. Analizamos la situación de las MSH en relación con la agenda de derechos humanos, como es el caso de la Declaración del Comité para la Eliminación de la Discriminación Contra la Mujer (CEDAW, en la sigla inglesa), donde los Estados Miembros dicen comprometerse a tomar medidas que garanticen a las mujeres el goce de derechos y libertades en igualdad de condiciones que los hombres. También revisamos algunas de las medidas adoptadas por el gobierno español. A continuación, se indaga en las narraciones de las participantes del estudio escogiendo de los relatos aquellas señales que nos indican aspectos constitutivos de dejación o vulneración de derechos, así como sus capacidades de resistencia. En este sentido, se toma como punto de referencia un poema escrito por María, mujer con experiencia en el sinhogarismo, participante en los talleres de teatro que se realizaron en los CA. Sin duda, esta creación constituye, en sí misma, un acto fundamental de derecho a contar y poder contarse (Ricoeur, 2005: 110). El poema dice así:

El grande y viejo espejo de madera: siempre allí, vigilante, indiscreto, cruel. Desafiante, observándome. Siempre atento. Yo intentaba esquivarlo en todos mis movimientos pero alguna que otra vez la curiosidad hacía que me viera a mí misma reflejada en él. Me acercaba lentamente, con miedo, con pudor. Lo miré y sí...era mi reflejo... pero...jesa no podía ser yo! No reconocí mis ojos, no reconocí mi piel. No reconocí tanta tristeza, soledad, agotamiento. No reconocí tanto vacío. El espejo relataba mi figura ganando la batalla con sus aliados: la violencia, el paro, la discriminación, la soledad. Tan solo tenía mi propia resistencia y la negación a verme así, de esa manera. Durante años ese espejo ganó miles de batallas. Yo gané la guerra. Ahora miro, lo miro, me miro sin miedos. (María, 14.11.2016, poema "Espejo”, recogido en el diario de campo de Manuel Muñoz-Bellerin)

\footnotetext{
${ }^{1}$ Los Centros de Acogida son centros residenciales de carácter temporal provistos por el sistema público español a través de los Servicios Sociales, que están dirigidos a personas sin hogar. Prestan diferentes servicios, principalmente alojamiento e higiene. Además, dependiendo de la tipología, también ofrecen asistencia psicosocial, orientación laboral, comedor, actividades de ocio y tiempo libre, etc. Pueden ser públicos, privados (gestionados por una $\mathrm{ONG}$ ) o concertados (de titularidad pública pero gestionados por una entidad privada).
} 
La poesía de María (Imagen 1) se considera un artefacto de investigación (Mannay, 2017) de carácter narrativo-creativo articulado dentro de los discursos de otras participantes acerca de las vulneraciones y las resistencias para el ejercicio práctico de los derechos humanos. Estas narraciones creativas se han obtenido a través del enfoque de la investigación basada en el arte (Barone y Eisner, 2012) que, en resumen, conjuga técnicas de las artes, en este caso el teatro, y las ciencias sociales. El artículo finaliza enunciando algunos de los aprendizajes derivados del proceso de investigación.

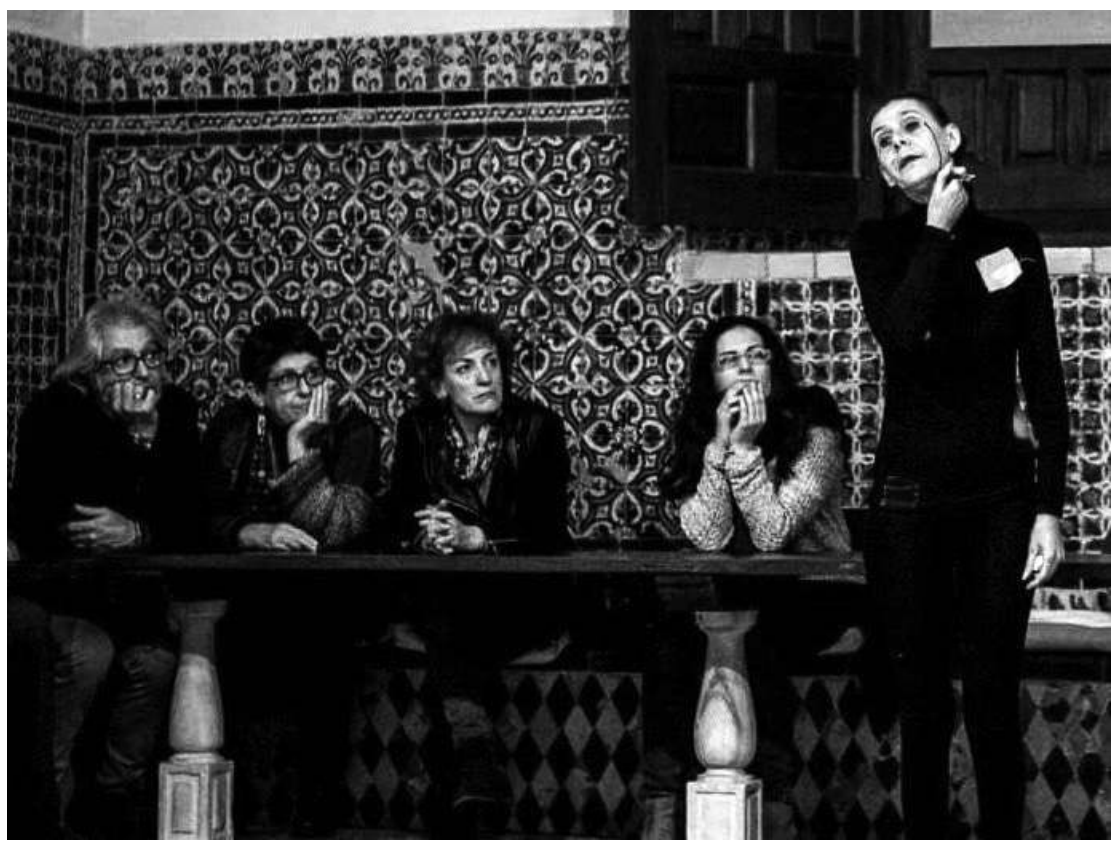

IMAGEN 1 - Performance de María recitando el poema "Espejo"

Nota: Fotografía captada el 29 de marzo de 2017, en las Jornadas de Reflexión “El valor social, educativo y cultural de las artes escénicas destinadas a la población infantil y adolescente", celebradas en Sevilla y organizadas por el Instituto de Cultura y la Asociación Te Veo.

Autoría: Asociación Te Veo.

1. Sinhogarismo y género: contextualización del problema en Europa y España El sinhogarismo en Europa es un problema social que abarca dimensiones tan complejas como el desempleo, la falta de vivienda y la ruptura de las redes sociofamiliares (Doherty, 2005). No sólo hace referencia a la carencia de vivienda digna, abarca otras situaciones estructurales de pobreza 
extrema y marginación, así como situaciones personales: carencia de vínculos familiares, enfermedad, pérdida del empleo, desprotección, ausencia de autoestima, victimización, etc., situando a las personas en una posición de subordinación y de exclusión social. Si bien el estado de bienestar proveyó de ciertas garantías en el equilibrio social y económico de sociedades como la española, la crisis económica del 2008 provocó el derrumbe del sistema de garantías atacando gravemente el empleo y la vivienda pública (Navarro et al., 2011). Otro de los efectos de la crisis fue el deterioro de las redes sociofamiliares que hasta entonces cumplieron la función de sostén tradicional, fundamentalmente en zonas de exclusión (Castel, 1995). Todos estos efectos tuvieron consecuencias devastadoras para las mujeres más vulnerables (Lombardo y León, 2014).

A los factores enunciados anteriormente, comunes a todas las personas sin hogar (en adelante PSH), en el caso de las MSH hay que sumar la desigualdad que sufren por la violencia doméstica. Este dato, compartido por diferentes autores (Moss y Singh, 2015; Mayock y Bretherton, 2016) evidencia la conexión entre sinhogarismo y estructura patriarcal (Watson, 2000), incrementando la vulnerabilidad de las mujeres. No obstante, no hay que confundir violencia de género con violencia doméstica, ya que aunque ambos conceptos puedan tener efectos semejantes, connotan diferencias. Si bien la primera toca de manera directa a la mujer, la violencia doméstica hace referencia al ámbito familiar. Para Maqueda Abreu (2006: 6), es necesario distinguirlas pues,

esa confusión de etiquetas, a veces interesada, entre violencia de género y violencia doméstica, contribuye a perpetuar la probada resistencia social a reconocer que el maltrato a la mujer no es una forma más de violencia, que no es circunstancial ni neutra sino instrumental y útil en aras de mantener un determinado orden de valores estructuralmente discriminatorio para la mujer.

Es importante tener en cuenta esta distinción para comprender que la violencia ejercida por el hombre dentro de la familia va dirigida a la integridad física y psíquica de la mujer como sujeto y no de manera exclusiva como rol dependiente del sistema familiar. De hecho, en el caso del sinhogarismo de género ${ }^{2}$ ambos tipos de violencia son formas enraizadas de un sistema de "violencia directa (visible) e indirecta (cultural y estructural)" (Galtung, 1998: 18).

\footnotetext{
${ }^{2}$ Denominamos así a la especificidad del sinhogarismo que atañe a la mujer y que tiene una determinada connotación de discriminación.
} 
En España, la violencia doméstica como origen del sinhogarismo provoca la salida del hogar y la separación de los hijos. Este hecho viene precedido de un elemento cultural con una gran carga social: al rol tradicional de la mujer como cuidadora y sustentadora del sistema familiar se le añade el lastre de ser madres susceptibles de no cuidar de sus hijos. A la violencia doméstica, se suma la violencia simbólica que la sociedad ejerce como respuesta ante el hecho del "abandono" del hogar familiar. Se la despoja de los atributos sociales y morales asignados por el sistema patriarcal como cuidadora (madre y cónyuge), una vez que ha tenido que salir del hogar, acentuando la estigmatización. Las opciones de una salida digna del hogar y de una mínima perspectiva de futuro pasan por el apoyo de sus redes familiares o amigos. Si se dan las condiciones idóneas, la primera elección para la mujer será recurrir a la ayuda de personas de confianza. No obstante, este tipo de apoyo comporta varios hándicaps, siendo uno de ellos que la ayuda ofrecida por la red extensa sea discontinua y fluctuante, sin asegurar una base con la que asentarse hacia un proyecto de vida futura. Otro problema es que la persona pase a vivir en un domicilio ajeno y de manera ocasional. En estos casos se produce una carencia de vivienda encubierta no reconocida en los estudios sociales (Pleace y Bretherton, 2013). A su vez, la domiciliación encubierta acarrea la invisibilidad del problema, pues es bastante probable que mientras dure el auxilio de la red la mujer no realice ninguna demanda a los Servicios Sociales, impidiendo que estos la apoyen en el ejercicio de sus derechos.

Respecto a los Servicios Sociales, tanto en España como en otros países europeos (Suecia, Reino Unido o Hungría) existe la dificultad de conceptualizar, diagnosticar y ofrecer atención de forma específica a las MSH (Moss y Singh, 2015). La falta de diagnóstico genera una doble paradoja. De un lado, muchas mujeres que salen del hogar por efecto de la violencia y el abuso doméstico son atendidas por los Servicios Sociales como víctimas de violencia de género. Del otro lado, en los CA no existe un protocolo específico para la detección de casos de violencia de género y doméstica. En tales ocasiones, las mujeres que demandan los recursos y servicios para PSH no son registradas como víctimas de violencia y son tratadas como el resto de los usuarios, siendo en muchas ocasiones las propias instituciones las encubridoras del problema. En cualquiera de los dos supuestos, no existe un análisis multidimensional de las circunstancias que rodean a las MSH. Esta es una de las razones de la necesidad de una mayor proliferación de estudios que profundicen en los factores macro y micro que acontecen en el sinhogarismo de género (Mostowska y Sheridan, 2016).

La naturaleza oculta del sinhogarismo en las mujeres, a la que se refiere Baptista (2010: 163), hace que resulte imprescindible la incorporación de 
la perspectiva de género en el campo del sinhogarismo. Así se demuestra en el estudio realizado en varios países europeos, realizado por Moss y Singh en 2015. Según esta investigación, los recursos para MSH no están especializados profesionalmente a nivel institucional y técnico. Tanto los recursos institucionales como las investigaciones científicas sobre sinhogarismo conceden a los hombres un papel hegemónico. Según Fabian (2016), esta situación de desigualdad fue advertida por el Parlamento Europeo, solicitando a los Estados Miembros una mayor actividad en el campo social y científico que analice las causas y efectos de la falta de hogar en las mujeres, así como las soluciones integrales al problema. Por otra parte, desde las últimas décadas hay autoras (Mayock y Bretherton, 2016) que avisan de la necesidad de visibilizar a las MSH dotándolas de mayor protagonismo (Williamson et al., 2014). Si bien los apoyos estatales dirigidos a procesos de empoderamiento de las PSH (Anderson, 2010) son escasos, en el caso de las MSH resultan apenas apreciables.

El incremento de las mujeres en el sinhogarismo es un síntoma más de la discriminación de género, reforzada por la condición de opresión y dominación (Young, 2000). La triple discriminación de la MSH (mujer, víctima de violencia y sin hogar) y el desigual acceso a las garantías sociales pone de manifiesto el androcentrismo social y cultural dominante. Uno de los impedimentos de este tipo de dominación es la indiferencia ante el reconocimiento de los derechos de las MSH. Frente al silencio y el ocultamiento social e institucional en el que se encuentran es necesario un reconocimiento sociopolítico como ciudadanas en igualdad; así se postula en tratados internacionales, como es el caso de la CEDAW o la Conferencia de Beijing. En el siguiente apartado revisamos los avances de las políticas públicas cotejándolos con las experiencias de las participantes, recogidas en sus discursos durante la investigación. Estos fragmentos permiten visibilizar algunas de las vulneraciones a las que son sometidas cotidianamente las MSH.

\section{De los derechos reconocidos por las políticas públicas a las narraciones de las protagonistas}

En este apartado examinaremos algunos de los derechos a los que se pueden acoger las MSH, poniéndolos en diálogo con fragmentos de los relatos de las participantes en la investigación. Para comenzar, en España partimos de la ausencia de una normativa específica que establezca garantías para las MSH. Esto da lugar a graves consecuencias en la vida de estas mujeres, tanto para las que sienten peligrar su seguridad y necesitan urgentemente salir de la vivienda familiar, como para aquellas que ya están fuera de la misma y no tienen las garantías de una vivienda digna. En el ámbito de la legislación internacional, 
la CEDAW (en vigor desde 1981) es un referente de antidiscriminación, ya que contempla una diversidad de cuestiones que afectan de forma directa a las mujeres a lo largo de los 30 artículos que componen el texto. Al mismo tiempo, señala a los Estados como responsables de eliminar la discriminación histórica que vienen padeciendo las mujeres, promoviendo para ello la igualdad sustancial entre los géneros. Es por esto que la CEDAW alienta a los países que la suscriban a que modifiquen las leyes que impidan el alcance de los derechos fundamentales por parte de las mujeres, así como a que introduzcan mecanismos afirmativos que permitan la defensa y promoción de la mujer. Entre estos se encuentra la provisión de una vivienda adecuada cuando sea necesario para eliminar cualquier situación discriminatoria que pueda sufrir la mujer (Bosh Meda, 2020).

La Cuarta Conferencia Mundial sobre la Mujer (en Beijing en 1995) supuso un avance en el reconocimiento de los obstáculos de las mujeres para tener acceso al derecho a la vivienda. Se acuerda, entre otras cuestiones, que los gobiernos tienen el deber de facilitar viviendas para las mujeres a precios razonables. Volviendo a España, los datos presentados en la Macroencuesta sobre violencia de género, efectuada por el Ministerio de Sanidad, Servicios Sociales e Igualdad en 2015, desvelaban que el $12,5 \%$ de las mujeres mayores de 16 años habían sufrido a lo largo de su vida violencia física o sexual de sus parejas o exparejas (Delegación del Gobierno para la Violencia de Género, 2015). Este porcentaje equivale a 2,5 millones de mujeres. Aunque en los últimos años los avances en la lucha contra la violencia de género han sido notables, aún queda camino por recorrer para erradicar las desigualdades y las situaciones de exclusión que viven numerosas mujeres. En el ámbito normativo, uno de los avances más significativos fue la aprobación de la Ley Orgánica 1/2004 de Medidas de Protección Integral contra la Violencia de Género ${ }^{3}$ que significó el reconocimiento jurídico del problema de la violencia sobre la mujer en España. Dicha ley tiene como objeto

actuar contra la violencia que, como manifestación de la discriminación, la situación de desigualdad y las relaciones de poder de los hombres sobre las mujeres, se ejerce sobre éstas por parte de quienes sean o hayan sido sus cónyuges o de quienes estén o hayan estado ligados a ellas por relaciones similares de afectividad, aun sin convivencia. (Artículo 1)

\footnotetext{
${ }^{3}$ Ley Orgánica 1/2004, de 28 de diciembre, de Medidas de Protección Integral contra la Violencia de Género, Boletín Oficial del Estado, número 313, de 29/12/2004. Consultado el 03.09.2021, en https://www.boe.es/eli/es/lo/2004/12/28/1/con.
} 
Uno de los puntos más destacados de esta ley radica en la garantía de derechos a las víctimas facilitándoles información, asistencia social integral y contribuyendo "a hacer reales y efectivos sus derechos constitucionales a la integridad física y moral, a la libertad y seguridad y a la igualdad y no discriminación por razón de sexo" (Artículo 17). Si bien en España la Ley 1/2004 ha generado un punto de inflexión histórico en lo que se refiere a legislación vigente contra la violencia de género, aún falta activar medidas de carácter preventivo y paliativo que sean eficientes. Otro de los avances fue la entrada en vigor de la Ley Orgánica 3/2007 de 22 de marzo 4 para la igualdad efectiva de mujeres. La principal novedad que aporta esta ley es la prevención de conductas discriminatorias, así como el llamamiento a todos los poderes públicos para que subsanen situaciones de desigualdad de género. Sin embargo, estos avances se vieron mermados "a partir del 2008 como respuesta a la crisis económica y en el marco de un proyecto político neoliberal" (Lombardo y León, 2014: 14). La igualdad de género se va diluyendo en la ejecución de políticas no específicas como son la vivienda, el trabajo o la sanidad, subordinando a la MSH a un tipo de discriminación que le es impuesta. En este sentido, tal como alega Crenshaw (2012: 95), la subordinación interseccional es necesariamente intencional; de hecho, frecuentemente es consecuencia de la imposición de una carga que interactúa con otras vulnerabilidades preexistentes para crear más desempoderamiento.

Como se viene diciendo, la triple discriminación que sufren las MSH se manifiesta de forma explícita en la violencia que sufren en sus familias, obligándolas a salir del hogar. Una difícil decisión que se produce después de soportarla de forma continuada, tal como comenta A. (50 años): 5 "Fue muy duro, tuve que aprovechar un viaje que él hizo [refiriéndose a su cónyuge] para marcharme, no aguantaba más”. Este comentario pone de manifiesto la delicada decisión de dejar el hogar y el carácter furtivo de la situación. Para muchas de ellas, después del miedo aparece la autoculpabilización, lo que les lleva a una parcial pérdida de la personalidad hasta llegar a "no conocerse a sí misma” (R., 46 años). La situación se agrava cuando las garantías jurídicas y sociales que pueden protegerla son imprecisas y de difícil acceso. En alusión a ello, C. (47 años) comentó: "En ese momento en el que te sientes muy poca cosa como persona es cuando más necesitas de apoyos seguros".

\footnotetext{
${ }^{4}$ Ley Orgánica 3/2007, de 22 de marzo, para la igualdad efectiva de mujeres y hombres, Boletín Oficial del Estado, número 71, de 23/03/2007. Consultado el 19.10.2021, en https://www.boe.es/ eli/es/lo/2007/03/22/3/con.

${ }_{5}$ En adelante, las citas de las participantes del trabajo de campo que aparezcan en el artículo serán indicadas por la inicial del nombre de la mujer y, entre paréntesis, su edad.
} 
Esta discriminación se ve acrecentada una vez que la mujer está en la calle y tiene que recurrir a la protección de los Servicios Sociales. En la ciudad de Sevilla, tan solo el 15,28 \% de las plazas de los CA están destinadas a mujeres. "La realidad es que hay más plazas de hombres que de mujeres, nosotras somos una minoría”, confirma M. L. (54 años) señalando claramente la masculinización de un espacio que les es ajeno. En el ámbito de la atención, las mujeres suelen ser percibidas como un usuario más donde "no se respeta lo que decimos", apunta C. (56 años); ni "tampoco lo que sentimos", añade K. (53 años). La no distinción del género en la intervención social con PSH potencia mayor nivel de dominación, dándose "condiciones institucionales que impiden a la gente participar en la determinación de sus acciones o de las condiciones de sus acciones" (Young, 2000: 68). Tras la violencia y la difícil decisión de la salida del hogar, las mujeres que no tienen recursos informales y acuden a la institucionalización se encuentran con este tipo de dominio impuesto (Roitman, 2011) que incumbe a la pérdida parcial de privacidad, al anonimato y a la alienación.

Volviendo a la Ley $1 / 2004$, es importante precisar la insistencia que se hace respecto a "la especialización de los colectivos profesionales que intervienen en el proceso de información, atención y protección a las víctimas" (Artículo 2). Inclusive, la Ley menciona claramente que dicha atención esté regulada desde un componente multidisciplinario (Artículo 19). Por el contrario, algunas de las participantes alegaron sentirse en un ámbito de opresión que para Young consiste

en procesos sociales institucionalizados que anulan la capacidad de las personas para interactuar y comunicarse con otras o para expresar sus pensamientos y perspectivas sobre la vida social en contextos donde otras personas pueden escucharlas. (2000: 68)

Para T. (39 años) esto se traduce en que "al igual que fuera [en alusión a la familia y la sociedad], aquí nadie respeta mis decisiones". Para F. (35 años), en estas instituciones no hay "un ambiente de apoyo, ni siquiera de mínima comprensión de nuestros problemas". Sin embargo, para E. (41 años) este tipo de prácticas institucionales van más allá, "es la ley la culpable de que no se nos trate con dignidad". En cuanto a la atención, A. (54 años) hace alusión a un modelo centrado "en el conformismo y la resignación con el que quieren acomodarnos a las circunstancias". Para mujeres como M. A. (59 años), con algunos años deambulando por los CA, hay momentos en que el abatimiento se instala "porque me siento culpable y siento tanta vergüenza que no quiero ver a nadie ni que me vean" (Imagen 2). Al dolor de perder un hogar, la separación de la familia, 
de vivir con recuerdos cargados de violencia e ignominia, se une el estado de "abandono" (P., 56 años) y "desconsuelo" (K., 33 años). Sentimientos estos que alejan cualquier atisbo de esperanza cuando se las invita a luchar por sus derechos. Palabras reiteradas en los discursos como "miedo", "violencia" y "dolor" están muy presentes en una cotidianeidad donde el horizonte de los derechos humanos es inexistente (Imagen 3).

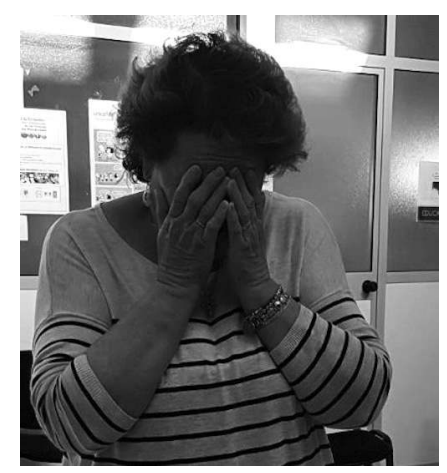

IMAGEN 2 - “No quiero ver a nadie". Fotografía que recoge algunos de los sentimientos y estados de ánimo de mujeres sin hogar

Autoría: Manuel Muñoz-Bellerin.

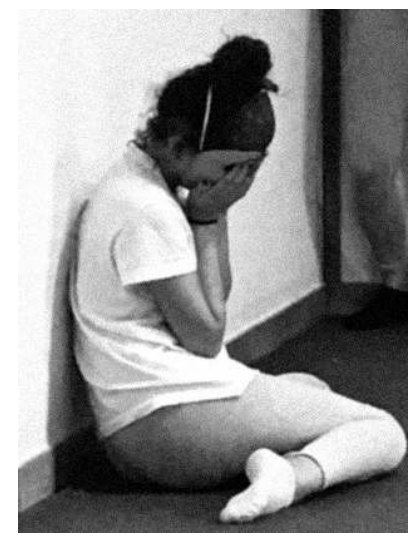

IMAGEN 3 - Fotografía que recoge el miedo sentido por una participante en uno de los talleres

Autoría: Manuel Muñoz-Bellerin. 
El espejo como objeto de autorrepresentación en el poema de María (51 años) es un símbolo de la falta de dignidad que ella sentía. Después de oír el poema, B. (44 años) exclamó: "Yo tampoco me miraba en el espejo porque no era yo, me daba miedo". Por su parte, M. J. (28 años) se cuestionaba a sí misma: "Ante el espejo de la vida, ¿cómo nos vemos a nosotras mismas?”. La respuesta de F. (50 años) aludía a estrategias que tienen que ver con la supervivencia: "Acostumbrándote a sufrir". Para algunas, el tiempo de estancia en los CA genera falta de seguridad y una ansiedad vital traducida en la necesidad de escapar de un sistema que reproduce la violencia machista: "Cuanto antes salga de aquí [CA] mejor", responde P. (29 años). Sin embargo, algunas reconocen que una salida en falso (del hogar al CA y de este al ámbito de la calle) aumentará las probabilidades de cronificación dentro del sinhogarismo, del cual es difícil de salir porque "no te facilitan los medios para que salgas adelante" (M. R., 44 años).

Frente a la opresión y la dominación expresadas por estas mujeres, Young (2000) plantea la resistencia en el autodesarrollo y la autodeterminación. Autodesarrollo como un ejercicio de las capacidades singulares y propias que cada mujer posee y que tiene el derecho de expresar como un rasgo de su identidad. Autodeterminación a través de la participación activa, articulada por medio de las decisiones y determinaciones que cada mujer asume como ciudadana. Ambas categorías hacen mención a la emancipación y quedan inscritas en el $3^{\text {er }}$ Artículo de la CEDAW, cuando apunta al desarrollo y progreso de la mujer con el objetivo de garantizarle el ejercicio y el goce de los derechos humanos y las libertades fundamentales en igualdad de condiciones con el hombre. Esto pasa por una comprensión de aquellos contextos donde se vulneran los derechos de las mujeres, así como la participación como principio democrático y del ejercicio de los derechos en su máxima expresión. En el siguiente apartado se reconoce la importancia del abordaje de las capacidades, como posibilidad de ir generando condiciones de dignidad, abriendo espacios para el reconocimiento de sus derechos. Para ello, se presentan narraciones que forman parte de un proceso de investigación-acción participativa que tiene como fundamento la transdisciplinariedad entre arte y ciencias sociales (Muñoz-Bellerin y Cordero-Ramos, 2019) y tiene com $\neq$ o referente la conexión del teatro aplicado.

\section{El desarrollo de las capacidades a través del teatro aplicado. Una pro- puesta para garantizar los derechos humanos de las mujeres sin hogar}

Una de las maneras posibles de garantizar el ejercicio de los derechos humanos que tienen las MSH es partiendo del pleno desarrollo de sus capacidades. 
Para Nussbaum, el enfoque de las capacidades está "comprometido con el respeto a las facultades de autodefinición de las personas" (2012: 38). Básicamente, las capacidades están imbricadas con las libertades sustanciales (Sen, 2010) que Nussbaum denomina como capacidades combinadas. Estas corresponden a una adecuación de las capacidades internas de cada sujeto, es decir, a "los rasgos y aptitudes entrenadas y desarrolladas [...] en interacción con el entorno social, económico, familiar y político" (Nussbaum, 2012: 41). Reivindicar los derechos tiene como objetivo su recuperación o conquista y esto pasa por visibilizar, señalar, los obstáculos que lo dificultan. Siguiendo a Gallardo (2009: 65), "los fundamentos de derechos humanos deben buscarse en testimonios y esto quiere decir en experiencias sociales históricamente situadas". Es la persona quien se siente con la capacidad de hablar (contar) a los demás, pero también de contarse a sí misma en un ejercicio de autoproducción (ibidem: 45), de un producirse a sí misma en movimiento con una identidad recobrada a través de la memoria de los cuerpos y las experiencias. En alusión a Spivak (2002), en el caso de la MSH se trataría de contraponer la posición de subalterna asignada por el sistema hegemónico dominante, enunciándose a sí misma y al resto del mundo en un gesto de lucha por la emancipación.

En cuanto a las iniciativas para garantizar la efectividad de los derechos humanos, las instituciones no tienen en cuenta las opiniones de las MSH. Este hecho se fue constatando a lo largo de la investigación. La mayoría de las participantes declararon no tener opciones ni oportunidades reales para decidir sobre su futuro inmediato. Las limitaciones para tomar decisiones es una constante en sus vidas, antes de ingresar en los CA y durante la estancia en estos. Esto les provoca miedo y las lleva, en ocasiones, a un cuestionamiento vital, "¿qué hacemos con nuestra vida: hacia dónde vamos?" (M. L., 39 años), reflejado en una corporalidad reducida a un "no soy nada" (L., 33 años) o a una pérdida del ser que se desliza en esta expresión: "He dejado de ser yo" (R., 61 años). Por el contrario, la elección de una metodología participativa basada en el protagonismo fue, sin duda, lo que facilitó que pudieran comenzar narrando sus sentimientos y sus vivencias para, posteriormente, recoger de forma colectiva sus reivindicaciones. Una participación que para Nussbaum (2012:54) está conectada con las "decisiones políticas que gobiernan nuestra vida; tener derecho a la participación política y a la protección de la libertad de expresión y de asociación". Participación que es, en definitiva, una acción frente a imposiciones efectuadas desde patrones de conductas y prácticas institucionalizadas ejercidas como medios de dominación. El alcance protagónico de las mujeres participantes en los talleres entre 2016 y 2019 tuvo un elemento central en las narrativas (Bruner, 2009) 
como alternativa al silencio impuesto a lo largo de sus vidas. No fue fácil. A los antecedentes de violencia doméstica y de género, había que añadirle la enorme dificultad que entrañaba un ambiente institucional dominado por hombres. La recuperación de las voces y las palabras supuso un proceso paulatino de reidentificación y, además, se fue convirtiendo en una forma de apropiación (Foucault, 2014) de un discurso que les permitía enunciarse y reconocerse a sí mismas. La agencialidad vista como acción y hecho político a través del hablar y del contar, tuvo como eje central el taller de teatro alrededor del cual se articularon dos espacios discursivos: oral y corporal (Diagrama 1).

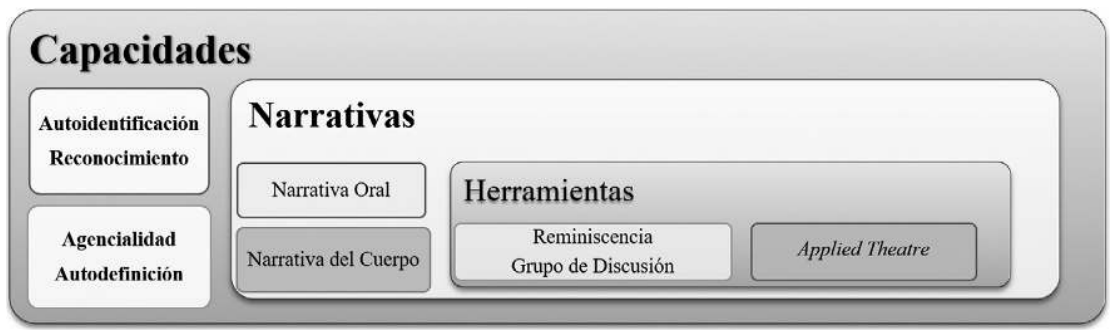

DIAGRAMA 1 - Metodología y esquema de las capacidades

Fuente: elaboración propia.

En los relatos surgió la posibilidad de trabajar la reminiscencia (Bornat, 2001) a través de las narraciones colectivas generadas en los grupos de discusión (Ibáñez, 2003). Por su parte, el cuerpo tomó protagonismo a través del teatro aplicado. La reminiscencia consiste en una actividad donde las personas "se comunican y comparten sus recuerdos con el propósito de comprenderse entre sí o de comprender una situación compartida" (Bornat, 2001: 57). Si bien esta autora hace referencia a la reminiscencia como una técnica fundamentalmente grupal, en nuestro caso se utilizó de forma individual permitiendo que las mujeres pudieran ir reconstruyendo pequeños fragmentos de sus vidas. A modo de microrrelatos, estos fragmentos eran un medio para cambiar el contenido del discurso entendiendo la dureza de narrar "un pasado que duele" (I., 55 años). La posibilidad de poder expresar "sentimientos de incomprensión" (como el caso de M., 64 años), se convirtió en un vehículo útil para el desahogo de emociones negativas contenidas. Los relatos fueron concebidos como un espacio biográfico donde cada protagonista mostraba su capacidad de narrar los acontecimientos de su vida, pasada o presente, eligiendo los sucesos o hitos que cada participante consideraba de especial relevancia en aquel momento. 
Al mismo tiempo, al poder narrarse de forma grupal se favoreció la creación de un discurso coral. Siendo el grupo, para Ibáñez (2003: 126), un "lugar privilegiado para la lectura de la ideología dominante", las participantes pudieron expresar problemas comunes como la violencia doméstica ejercida por "los hermanos, padres y maridos" (B., 44 años). Los temas de interés elegidos por las participantes en los grupos de discusión fueron la violencia, la opresión, la discriminación o la desigualdad. Poder contar cómo se sentían junto a las otras compañeras les permitió expresar capacidades como empatía, apoyo mutuo y cooperación. Para ello, sin duda, el teatro aplicado fue el mejor aliado para mostrar las capacidades creativas. Para Prendergast y Saxton (2009), más que una técnica, se trata de una serie de prácticas alternativas de carácter teatral que vienen realizándose desde hace décadas en todo el mundo a través de otros conceptos como teatro popular, teatro social, teatro del oprimido, etc. Para estas autoras, "teatro aplicado ha surgido como término paraguas que engloba todos estos términos y prácticas previos" (ibidem: 7 ; traducción de los autores). La aplicación metodológica del teatro aplicado es un modo de producción participativa (Mannay, 2017) al potenciar el empoderamiento de colectivos (Prentki y Pammenter, 2014) como fue el caso de las MSH. De manera más específica, las técnicas más utilizadas fueron teatro imagen (Boal, 1989) y gesto social (Brecht, 2004). Ambas ahondaron en la expresividad corporal como signo y significado en el contexto de la opresión patriarcal ejercida sobre las participantes, tanto por parte de la sociedad como por las instituciones. Aludiendo a esto último, K. (46 años) comentó que "con el teatro puedo romper las cadenas de las incomprensiones, de ser señalada, de la soledad y dejar de ser una mujer invisible". De esta forma el teatro les ofreció la posibilidad de expresar con sus cuerpos vivencias, emociones, anhelos y, con ello, mostrarse como quieren que se las vea en sus vidas cotidianas.

El teatro imagen posibilitó la representación de conflictos vividos por las participantes a través de la producción de tres tipos de secuencias. En la primera, se alude a la imagen real en la que las actuantes esculpen los cuerpos de las compañeras con la finalidad de representar el conflicto enunciado. Posteriormente esas imágenes se convierten en imágenes ideales que escenifican soluciones diversas a la imagen real precedente. Finalmente, se analizan y proponen ambas para convertirlas en imágenes en tránsito que señalan, de manera didáctica, cómo efectuar los cambios necesarios entre la realidad y el deseo. El análisis del gesto social permitió evidenciar los signos marcados en los roles y las posiciones de género asumidas a lo largo de sus vidas, que recreaban interacciones cargadas de elementos donde se vulneran cotidianamente sus derechos. A través de las capacidades creativas 
fue aflorando el reconocimiento de un uso políticosocial de la palabra y el cuerpo como mecanismos discursivos. En las distintas sesiones del trabajo de campo germinaron voces de esperanza como la de R. (61 años): "cada día tenemos que estar superándonos y por eso lo que digan los demás [por la sociedad] no nos afecta; cada día es una superación"; o como añade L. (33 años): "tenemos nuestra dignidad, no tenemos que echar cuenta de cómo nos miran". Como colofón, muchas de estas narraciones sirvieron para la creación de performances construidas por ellas mismas y escenificadas en diversos espacios culturales de la ciudad de Sevilla, durante los años que duró la investigación.

\section{Conclusiones}

Tanto la literatura académica como las narraciones de las mujeres participantes en la investigación muestran cómo en sociedades democráticas continúan sucediendo violaciones de derechos humanos, como así pone de manifiesto el fenómeno del sinhogarismo en la mujer. En base al análisis realizado, se evidencia que, aunque en España exista un avance en las políticas públicas para la igualdad de las mujeres, estas no terminan de materializarse en el caso de las MSH, que siguen sufriendo de forma más evidente (que los hombres) las consecuencias de la exclusión social. La pandemia del COVID-19 ha empeorado aún más la situación. El confinamiento obligatorio en las instituciones de acogida o las restricciones de los contactos con las redes de apoyo externas, unido al decremento en las políticas sociales en materia de género y sinhogarismo, ha generado aún más, si cabe, una nueva forma de dominación. Una dominación que tiene como efecto una mayor privación de derechos.

Desde el punto de vista empírico, resultan escasas las investigaciones que profundizan en esta temática. Conviene resaltar los trabajos que vienen haciendo redes como Women's Homelessness in Europe Network (WHEN), ${ }^{6}$ que cuenta con la colaboración de investigadores, legisladores y profesionales con interés en la falta de vivienda de las mujeres. Otra investigación empírica a destacar es la realizada por las autoras Mayock y Sheridan (2016). Ambas autoras muestran el alto nivel de inseguridad y miedo sentido por las entrevistadas, tanto a nivel institucional como social, así como el pesimismo que muchas manifiestan acerca de las perspectivas de conseguir una vivienda estable. Este tipo de estudios están sacando a la luz las consecuencias del sinhogarismo para las mujeres en Europa. En el caso español prevalece la invisibilidad de la situación en los estudios realizados en la última década. Estos apenas reconocen las particularidades de las MSH,

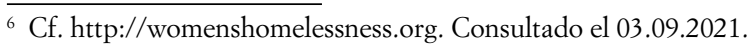


que permanecen ocultas bajo las generalizaciones de las categorías utilizadas. Al mismo tiempo, esta invisibilidad es compartida en las actuaciones dentro de los CA, donde los dispositivos de intervención no tienen en cuenta la perspectiva de género a la hora de diseñar y ejecutar estrategias y programas para este tipo de mujeres.

Una dificultad añadida es que en los CA, que han formado parte de esta investigación, las propias mujeres se resisten a participar en actividades o espacios donde el número de hombres es muy superior. Para lograrlo, se hace necesario ir generando estrategias paritarias que rompan con los modelos de relaciones jerarquizados aún persistentes y enraizados a patrones y códigos patriarcales de peso. Estos códigos están sumidos en lo que Young denominó como imperialismo cultural que "consiste en hacer que un grupo sea invisible al mismo tiempo que resulta marcado y estereotipado" (2000: 208). Esto se hace patente en los contextos de subordinación donde más se perpetúan los procesos de jerarquización e interdependencia (Crenshaw, 2012) y en aquellos donde los niveles de subsistencia son más extremos, como es el caso del sinhogarismo. En estos entornos, es urgente impulsar estrategias para el desarrollo de las capacidades que contribuyan a visibilizar la realidad de las MSH y desestructurar elementos de dominación recogidos en las narraciones de las propias protagonistas.

Resulta imprescindible la sensibilización social, un mayor conocimiento a través de las investigaciones académicas, así como un mayor impulso de programas sociales que incorporen metodologías participativas basadas en las artes. Este tipo de metodologías requieren una especialización profesional, ya que no consiste en un ejercicio profiláctico de empoderar a un grupo de mujeres desde la trivialidad y futilidad de un espectáculo teatral. Tampoco se trata de una representación artística, sin más. La utilización de la representación teatral o la performance (en su variante reivindicativa) es una fase más dentro de un proceso complejo que traspasa la individualidad corporal para ir construyendo de manera abierta una creación colectiva. Palabras y cuerpos expresaron y representaron, desde otros modos vivenciales, una territorialidad propia, constructora de afectos y cuidados, reflexiva y crítica, diferente a otros modelos donde predominan la estereotipia y la estigmatización. En los talleres el cuerpo se convirtió en vehículo para el relato sociopolítico de denuncia de la violencia doméstica y de la violencia de género, a partir de las experiencias vividas por las participantes. Esta puede ser una vía para que los derechos humanos sean una proximidad y no queden en la esperanza (Sikkink, 2018). Si bien no se obvia el trabajo de algunas instituciones en la defensa de los derechos, en esta investigación las mujeres demostraron, por medio de sus testimonios y acciones, lo imprescindible que resulta la 
participación activa a través de lo que Herrera Flores definió como proceso cultural de los derechos humanos, es decir, modos de "reaccionar frente el entorno de relaciones en los que viven" (2008: 40).

Para finalizar, la crueldad del espejo al que hace referencia María, la autora del poema que encabeza este artículo, es la alegoría de la proyección que emana del sistema hegemónico patriarcal, desafiante para las mujeres que se encuentran en situación de exclusión. En el mismo, la artista alude claramente a su rostro desfigurado "por la violencia, el paro, la discriminación y la soledad", según sus propias experiencias, compartidas en uno de los foros de debate tras la performance representada en una sala teatral de la ciudad de Sevilla. Sin embargo, a pesar de todo, María resistió: "tuve aún fuerzas, energías para afrontar todo eso". Consiguió "salvarse para contarlo", recuperarse como mujer y narrarlo de forma artística para que sirva de reconocimiento y de reivindicación. El poema invita a mirarnos en el mismo espejo para reconocer sin miedo nuestras vulnerabilidades como sociedad. Para ganar la guerra de la discriminación es necesario mirar de frente y reconocer las condiciones de indignidad que viven las $\mathrm{MSH}$, no solo para comprender como se sienten y acompañarlas en sus batallas cotidianas, sino para generar una nueva lógica donde la paridad esté presente en cada uno de los espacios de sociabilidad que habitamos.

Revisado por José Morales

\section{Referencias bibliográficas}

Anderson, Isabel (2010), "Services for Homeless People in Europe: Supporting Pathways out of Homelessness", in Eoin O'Sullivan; Volker Busch-Geertsema; Deborah Quilgars; Nicholas Pleace (comps.), Homelessness Research in Europe. Bruxelles: FEANTSA, 41-64. Arfuch, Leonor (2018), La vida narrada. Memoria, subjetividad y politica. Córdoba: Eduvim. Baptista, Isabel (2010), "Women and Homelessness", Homelessness Research in Europe, 4(1), 163-185.

Barone, Tom; Eisner, Elliot (2012), Arts Based Research. California: Sage Publications. Boal, Augusto (1989), Teatro del oprimido. México: Nueva Imagen. Traducción de Graciela Schmilchuk [ed. orig. 1980].

Bornat, Joanne (2001), "Reminiscencia e historia oral: ¿universos paralelos o empeño común?”, Revista Historia, Antropología y Fuentes Orales, 26, 53-76. Traducción de Mireia Bofill.

Bosh Meda, Jordi (2020), "El derecho de la mujer a la vivienda en España (2008-2017)", Revista de Estudios Feministas, 28(1), e54148. Consultado el 22.08.2021, en https:// doi.org/10.1590/1806-9584-2020v28n154148. 
Brecht, Bertolt (2004), Escritos sobre teatro. Barcelona: Alba Editorial. Traducción de Genoveva Dieterich [ed. orig. 1933-1947].

Bruner, Jerome (2009), Actos de significado. Más allá de la revolución cognitiva. Madrid: Alianza Editorial. Traducción de Beatriz López [ed. orig. 1986].

Castel, Robert (1995), "De la exclusión como estado a la vulnerabilidad como proceso", Archipiélago: Cuadernos de Crítica de la Cultura, 21, 27-36.

Crenshaw, Kimberlé Williams (2012), “Cartografando los márgenes: interseccionalidad, políticas identitarias, y violencia contra las mujeres de color”, in Raquel (Lucas) Platero (coord.), Intersecciones: cuerpos y sexualidades en la encrucijada. Barcelona: Bellaterra, 87-122. Traducción de Raquel Lucas Platero y Javier Sáez.

Doherty, Joe (2005), "El origen del sinhogarismo: perspectivas europeas", Documentación Social, 138, 41-61. Traducción de Kate Angus.

Delegación del Gobierno para la Violencia de Género (2015), Macroencuesta de violencia contra la mujer 2015. Madrid: Ministerio de Sanidad, Servicios Sociales e Igualdad. Consultado el 02.09.2021, en http://www.violenciagenero.igualdad.mpr.gob.es/ violenciaEnCifras/macroencuesta2015/Macroencuesta2015/home.htm.

Fabian, Dalma (2016), "Perspectives on Women's Homelessness", The Magazine of FEANTSA, Summer, 2-3. Consultado el 15.02.2021, en https://www.feantsa.org/download/summer-2016-perspectives-on-women-s-homelessness1684329503268833210.pdf.

Foucault, Michel (2014), Las redes del poder. Buenos Aires: Prometeo. Traducción de Fernando Crespo [ed. orig. 1968].

Gallardo, Helio (2009), Derechos humanos como movimiento social. Bogotá: Ediciones desde abajo.

Galtung, John (1998), Tras la violencia. 3R: reconstrucción, reconciliación, resolución. Bilbao: Bakeaz. Traducción de Teresa Toda.

Herrera Flores, Joaquín (2008), La reinvención de los derechos bumanos. Sevilla: Atrapasueños. Consultado el 03.09.2021, en http://www.derechoshumanos.unlp. edu.ar/assets/files/documentos/la-reinvencion-de-los-derechos-humanos.pdf.

Ibáñez, Jesús (2003), Más allá de la sociología. El grupo de discusión: técnica y práctica. Madrid: Siglo XXI.

Lombardo, Emanuela; León, Magarita (2014), "Políticas de igualdad de género y sociales en España: origen, desarrollo y desmantelamiento en un contexto de crisis económica”, Revista Investigaciones Feministas, 5, 13-35.

Mannay, Dawn (2017), Métodos visuales, narrativos y creativos en investigación cualitativa. Madrid: Narcea Ediciones. Traducción de Sara Alcina Zayas.

Maqueda Abreu, María Luisa (2006), "La violencia de género: entre el concepto jurídico y la realidad social”, Revista Electrónica de Ciencia Penal y Criminología, 8, art. 02, 1-13. Consultado el 03.09.2021, en http://criminet.ugr.es/recpc/08/recpc08-02.pdf. Mayock, Paula; Bretherton, Joanne (2016), Women's Homelessness in Europe. London: Palgrave Macmillan. 
Mayock, Paula; Sheridan, Sarah (2016), "Women and Long-Term Homelessness”, The Magazine of FEANTSA, Summer, 4-7. Consultado el 03.09.2021, en https:// www.feantsa.org/download/summer-2016-perspectives-on-women-s-homeless ness1684329503268833210.pdf.

Muñoz Bellerin, Manuel; Cordero Ramos, Nuria (2019), “Teatro, empoderamiento y capacidades: el teatro como herramienta de la acción social desde la ética de la representación”, Dilemata. Revista Internacional de Éticas Aplicadas, 29, 109-120.

Moss, Kate; Singh, Paramjitv (2015), Women Rough Sleepers in Europe: Homelessness and Victims of Domestic Abuse. Bristol: Policy Press.

Mostowska, Magdalena; Sheridan, Sarah (2016), "Migrant Women and Homelessness", in Paula Mayock; Joanne Bretherton (comps.), Women's Homelessness in Europe. London: Palgrave MacMillan, 235-263.

Navarro, Vicenç; Torres López, Juan; Garzón Espinosa, Alberto (2011), Hay alternativas. Propuestas para crear empleo y bienestar en España. Madrid: Sequitur.

Nussbaum, Martha C. (2012), Crear capacidades. Propuesta para el desarrollo bumano. Barcelona: Paidós. Traducción de Albino Santos Mosquera.

Pleace, Nicholas; Bretherton, Joanne (2013), "Measuring Homelessness and Housing Exclusion in Northern Ireland: A Test of the ETHOS Typology". Consultado el 16.05.2019, en https://www.york.ac.uk/media/chp/documents/2013/measuring homelessness_and_housing_exclusion_in_northern_ireland.pdf.

Prendergast, Monica; Saxton, Juliana (comps.) (2009), Applied Theatre: International Case Studies and Challenges for Practice. Bristol/Chicago: Intellect Ltd.

Prentki, Tim; Pemmenter, David (2014), "Living beyond Our Means, Meaning beyond Our Lives: Theatre as Education for Change”, Applied Theatre Research, 2(1), 7-19. Ricoeur, Paul (2005), Caminos del reconocimiento. Madrid: Editorial Trotta. Traducción de Agustín Neira [ed. orig. 2004].

Roitman, Marcos (2011), Democracia sin demócratas y otras invenciones. Madrid: Sequitur. Segato, Rita (2019), "Memoria, violencia y colonialidad". Trabajo presentado en el Coloquio Internacional "La memoria en la encrucijada del presente. El problema de la justicia”, 10, 11 y 12 de abril, Facultad de Ciencias Sociales, Universidad de Buenos Aires, Buenos Aires, Argentina.

Sen, Amartya (2010), La idea de la justicia. Madrid: Santillana Ediciones. Traducción de Hernando Valencia Villa [ed. orig. 2009].

Sikkink, Kathryn (2018), Razones para la esperanza. La legitimidad y efectividad de los derechos bumanos de cara al futuro. Buenos Aires: Siglo XXI. Traducción de Sebastián F. Villamizar Santamaría.

Spivak, Gayatri Chakravorty (2002), “¿Puede hablar la subalterna?”, Asparkia. Investigació Feminista, 13, 207-214. Traducción de M. Rosario Martín Ruano [ed. orig. 1999]. Consultado el 02.09.2021, en https://www.e-revistes.uji.es/index. php/asparkia/article/view/871. 
Watson, Sophie (2000), “Homelessness Revisited: New Reflections on Old Paradigms”, Urban Policy and Research, 18(2), 159-170.

Williamson, Emma; Abrahams, Hilary; Morgan, Karen; Cameron, Ailsa (2014), "Tracking Homeless Women in Qualitative Longitudinal Research", European Journal of Homelessness, 8(2), 69-91.

Young, Iris Marion (2000), La justicia y la política de la diferencia. Valencia: Cátedra. Traducción de Silvina Álvarez Medina [ed. orig. 1990].

\section{Nuria Cordero-Ramos}

Grupo de Investigación Social y Acción Participativa-GISAP, Universidad Pablo de Olavide de Sevilla I Red LIESS - Laboratorio Iberoamericano para el Estudio Sociohistórico de las Sexualidades Ctra. de Utrera, km. 1 41013, Sevilla, España

Contacto: ncroram@upo.es

ORCID: https://orcid.org/0000-0002-9875-1042

\section{Manuel Muñoz-Bellerin}

Grupo de Investigación Social y Acción Participativa-GISAP, Universidad Pablo de Olavide de Sevilla Ctra. de Utrera, km. 1 41013, Sevilla, España

Contacto:mfmunbel@upo.es

ORCID: https://orcid.org/0000-0003-0980-6847

Recibido: 06.05.2020

Aceptación comunicada: 03.09.2021

https://doi.org/10.4000/rccs.12407 


\section{Human Rights in front of the Mirror. Narratives of Homeless Women in the Exercise of Their Capacities}

Taking into account the consequences caused by homelessness in women and the recognition of human rights, this article looks at the experiences of homeless women living in shelters in the city of Seville (Spain). It is based on the accounts from the women themselves, thus representing a form of allegory for resistance. These narratives were recorded during a series of workshops, where the women were able to share their feelings and experiences individually and collectively through art. Our aim here is to show how applied theatre can be used as a creative tool to open up spaces which afford homeless women dignity and offer them the chance to show their skills to the rest of society.

Keywords: homelessness; host institutions; human rights violation; Spain; women's rights.
Les droits humains devant le miroir. Récits de femmes sans-abri dans l'exercice de leurs capacités

Partant des conséquences que le sans-abrisme provoque chez les femmes et ayant à l'esprit la reconnaissance des droits humains, on analyse les expériences des protagonistes elles-mêmes : des femmes sans-abri qui vivent dans des centres d'accueil de la ville de Séville (Espagne). On prend comme référence les récits des protagonistes elles-mêmes comme ressource allégorique de l'espace pour raconter des expériences de résistance. Ces récits ont été racontés lors de la réalisation d'une série d'ateliers où les femmes ont partagé des émotions et des expériences grâce à l'appropriation personnelle et collective du langage artistique. Ce travail vise à mettre en évidence la dimension créative du théâtre appliqué en tant qu'outil permettant de créer des espaces qui garantissent la dignité des femmes sans-abri, en leur offrant la possibilité de montrer leurs capacités à l'ensemble de la société.

Mots-clés: droits de la femme; Espagne; institutions d'accueil; SDF; violation des droits humains. 
